\title{
Congenital lobar emphysema: a rare cause for respiratory distress in newborns and infants
}

${ }^{\text {II. H. D. S Pradeep }}{ }^{1}$, Dhammika Rasnayaka ${ }^{1}$, Ridma Jayarathna ${ }^{2}$

${ }^{1}$ Department of Thoracic Surgery, National Hospital for Respiratory Diseases, Welisara.

${ }^{2}$ Department of Respiratory Medicine, Lady Ridgeway Hospital, Colombo.

Keywords: Congenital; lobar emphysema; respiratory; distress; newborn

\section{Introduction}

Congenital lobar emphysema is a rare disorder commonly presents with neonatal or infantile respiratory distress. The rarity and the associated cardiac abnormalities have made it a diagnostic challenge in paediatric practice. High degree of clinical suspicion aided with contrast enhanced computerized tomography of the chest (CECT) is the key to diagnosis. Prompt diagnosis with timely thoracic surgical intervention is essential to save affected lives.

\section{Case 1}

A four months old baby with ventricular septal defect (VSD) presented to a paediatric cardiac unit due to worsening respiratory symptoms. Two dimensional echocardio-graphy demonstrated a large muscular VSD with a significant left to right shunt and moderate pulmonary hypertension. Early surgical intervention was planned and poly tetrafluroethane patch repair of VSD performed with uneventful intraoperative and immediate post-operative period.

Progressive respiratory distress with diminished breath sounds on right chest was noted on 7th post operative day, however there wasn't any clinical and biochemical features of lower respiratory tract infection. Chest $\mathrm{X}$ - ray revealed diminished lung markings at the centre of the right hemi thorax with collapsed upper and lower lobes. Clinical suspicion of pneumothorax was made and re-evaluated by the thoracic team where the CECT thorax demonstrated the lobar emphysema of right middle lobe.

After fibre optic bronchoscopy (F.O.B) the baby underwent axillary thoracotomy under single lumen endotracheal tube intubation. Hyper expansion of right middle lobe with collapse of upper and lower lobes noted per-operatively and middle lobe resection done securing the pulmonary vessels to middle lobe and middle lobar bronchus. Baby was successfully extubated on second post-operative day.

\footnotetext{
Correspondence: I.H.D Saman Pradeep

E-mail: samaniddagoda@gmail.com

Received: 08-04-2018 Accepted: 19-07-2018

(iD) http://orcid.org/0000-0003-3526-1684

DOI: http://doi.org/10.4038/sljs.v36i3.8525
}

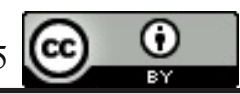

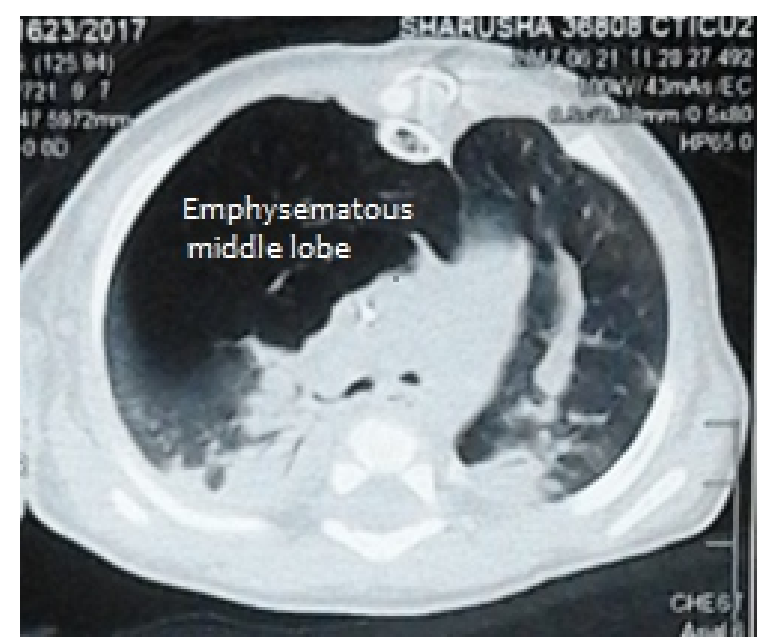

Figure 1. CECT chest demonstrating Middle lobe emphysema

\section{Case 2}

A six months old baby was investigated for cough, shortness of breath and fever for 1 week. A hyper lucent right lung was noted on Chest X-ray. CECT thorax revealed a hyperlucent anteromedial aspect of the right hemi thorax with mediastinal shift suggestive of either congenital lobar emphysema or congenital bullae involving right middle lobe. As the patient was progressive in symptoms thoracic team planned for the right thoracotomy and exploration.

Child was intubated with a single lumen endotracheal tube and underwent right axillary thoracotomy and exploration. The hyper expanded middle lobe compressing the upper and lower lobes was witnessed and middle lobectomy was performed after securing vascular pedicle and middle lobar bronchus. Rest of the surgical procedure was uneventful. Patient was extubated on the following day.

\section{Case 3}

A two months old baby girl transferred from a local hospital due to progressive shortness of breath, tachypnea, chest recessions and fever while managing as right upper lobar bronchopneumonia. Baby needed ventilator support for respiratory failure and the chest $\mathrm{X}$ ray revealed hyper lucent left lung fields with mediastinal shift to right side and inflammatory markings on right lung field. CECT revealed emphysematous left upper lobe with ipsilateral lower lobe collapse and right lower lobar consolidation and congenital 
lobar emphysema of left upper lobe. Thoracotomy and exploration was planned early as child could not come out of the ventilator. Externally compressed left upper lobe bronchus was noted on pre- operative F.O.B.

Left axillary thoracotomy was done with single lumen endotracheal intubation. On exploration, emphysematous left upper lobe with collapsed left lower lobe was found and left upper lobectomy done after securing pulmonary vessels and left upper lobar bronchus. Child had a protracted recovery due to coexisted bronchopneumonia of the right lung.

\section{Discussion}

Two major pathological forms been discussed in the CLE as hypo alveolar and poly alveolar depending on the number of over distended alveoli present in histopathological specimens compared with normal lung architecture. Abnormalities of the

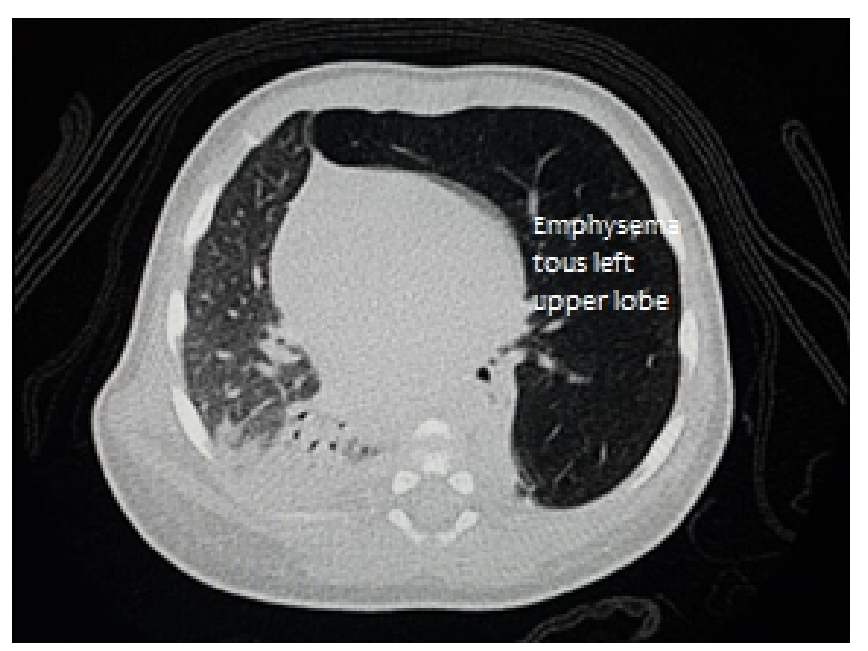

Figure 2. CECT chest demonstrating Left upper lobe emphysema.

bronchial cartilage development and resultant ball valve effect with air trapping, vascular abnormalities resulting bronchial stenosis and bronchogenic cysts and congenital cytomegalovirus infection have been postulated as possible etiopathogenesis of the condition [4]. In up to $15 \%$ of the cases there have been demonstrable congenital cardiac defects including VSD or patent ductus arteriosus [1,3].

Upper lobes have more predilections for involvement in CLE. Left upper lobe $(40-45 \%)$ is the most commonly affected preceded by right middle lobe $(30 \%)$ and right upper lobe $(20 \%)$. Hence right hemi thorax is the most commonly affected side [4].
Soon after the birth, the affected lobe is seen as homogeneously opaque region in chest $\mathrm{X}$ ray due to fetal lung fluid and distended lymphatic channels. Hyperlucency and diminished vascular lung markings with shifting of mediastinum and diaphragmatic depression are seen in established cases with clinically significant respiratory compromise. CECT of the chest is the investigation of choice for further anatomical details of the thorax in great detail.

Depending on the clinical presentation and radiological findings bronchial atresia with air trapping, congenital cystic adenomatous malformation (CCAM), congenital pulmonary airway malformation (CPAM), pulmonary arterial hypoplasia, pulmonary hypoplasia, Sawyer-James syndrome due to post infective obliterate bronchiolitis should be considered as the differential diagnoses.

Surgical resection of the affected lobe is the definitive management in CLE. The age of the child, clinical presentation, degree of respiratory compromise and associated heart disease are key factors to consider in timing of surgical intervention. In patients with cardiorespiratory compromise with mediastinal shift and compression of unaffected lobes necessitate lobectomy of affected segments. Histopathology of above mentioned cases revealed over distended alveoli confirming the diagnosis.

The diagnosis and management of CLE poses a great challenge upon clinicians in paediatric practice. High degree of clinical suspicion with timely thoracic surgical intervention can reduce associated morbidity and mortality.

All authors disclose no conflict of interest. The study was conducted in accordance with the ethical standards of the relevant institutional or national ethics committee and the Helsinki Declaration of 1975, as revised in 2000 .

\section{References}

1. Gordon I, DempseyJ.E. Infantile lobar emphysema in association with congenital heart disease. Clinical radiology. 41. 48-52. doi.org/10.1016/S0009-9260(05)80933-X.

2. Asok Kumar Datta,Syamali Mandal,Jadab Kumar Jana. Congenital lobar emphysema: a case report. Cases Journal 2009,2:67 doi:10.1186/1757-1626-2-67.

3. Dogan R, Dogan OF, Yilmaz M, Demircin M, Pasaoglu I, Kiper N, Ozcelik U, Boke E. Surgical management of infants with congenital lobar emphysema and concomitant congenital heart disease. Heart Surg Forum. 2004;7(6):E644-9. doi:10.1532/HSF98.20041041.

4. Ozçelik U, Göçmen A, Kiper N, Doğru D, Dilber E, Yalçin EG. Congenital lobar emphysema: evaluation and long-term followup of thirty cases at a single centre. Pediatr Pulmonol. 2003 May;35(5):384-91.doi:10.1002/ppul.10240.

\section{Learning Points:}

- Congenital lobar emphysema is a rare disorder which commonly presents as respiratory distress in newborns and infants.

- Timely thoracic surgical intervention can reduce morbidity and mortality associated with this condition. 